\title{
Solar neutrino oscillations and the recent results of BOREXINO and SNO
}

\author{
Michael Wurm* for the Borexino Collaboration ${ }^{\dagger}$ \\ Physikdepartment E15, Technische Universität München, James-Franck-Str., D-85748 Garching \\ E-mail: michael.wurmeph.tum.de
}

\begin{abstract}
The present contribution reviews the latest results of the solar neutrino experiments Borexino and SNO and their impact on the standard solar model and the global analysis of neutrino oscillations: The Borexino result on the ${ }^{7} \mathrm{Be}$ neutrino survival probability gives, for the first time, experimental evidence of vacuum neutrino oscillations at sub-MeV energies, backing up the MSW-LMA oscillation scenario. Nevertheless, the search for non-standard effects in the transition region from vacuum to matter-dominated oscillations in the energy regime from 1 to $5 \mathrm{MeV}$ is still ongoing: Both Borexino and SNO have by now published analyses of the ${ }^{8} \mathrm{~B}$ neutrino flux below $5 \mathrm{MeV}$ that provide mutually consistent but not conclusive results regarding oscillation models. The prospects of Borexino for an analysis of the ${ }^{8} \mathrm{~B}$ neutrino flux below the present threshold of $3 \mathrm{MeV}$ and for a first-time direct measurement of the pep neutrino line at $1.4 \mathrm{MeV}$ in energy will be outlined.
\end{abstract}

The Xth Nicola Cabibbo International Conference on Heavy Quarks and Leptons,

October 11-15, 2010

Frascati (Rome) Italy

*Speaker.

${ }^{\dagger}$ The Borexino Collaboration: G. Bellini, J. Benziger, D. Bick, S. Bonetti, M. Buizza Avanzini, B. Caccianiga, L. Cadonati, F. Calaprice, C. Carraro, A. Chavarria, A. Chepurnov, F. Dalnoki-Veress, D. D’ Angelo, S. Davini, A. Derbin, A. Etenko, F. von Feilitzsch, K. Fomenko, D. Franco, C. Galbiati, S. Gazzana, C. Ghiano, M. Giammarchi, M. GögerNeff, A. Goretti, E. Guardincerri, S. Hardy, Aldo Ianni, Andrea Ianni, M. Joyce, V. Kobychev, Y. Koshio, G. Korga, D. Kryn, M. Laubenstein, T. Lewke, E. Litvinovich, B. Loer, F. Lombardi, P. Lombardi, L. Ludhova, I. Machulin, S. Manecki, W. Maneschg, G. Manuzio, Q. Meindl, E. Meroni, L. Miramonti, M. Misiaszek, D. Montanari, V. Muratova, L. Oberauer, M. Obolensky, F. Ortica, M. Pallavicini, L. Papp, L. Perasso, S. Perasso, A. Pocar, R. S. Raghavan, G. Ranucci, A. Razeto, A. Re, P. Risso, A. Romani, D. Rountree, A. Sabelnikov, R. Saldanha, C. Salvo, S. Schönert, H. Simgen, M. Skorokhvatov, O. Smirnov, A. Sotnikov, S. Sukhotin, Y. Suvorov, R. Tartaglia, G. Testera, D. Vignaud, R. B. Vogelaar, F. von Feilitzsch, J. Winter, M. Wojcik, A. Wright, J. Xu, O. Zaimidoroga, S. Zavatarelli, and G. Zuzel 
The present contribution reviews the current status of solar neutrino oscillation physics. Starting from a short overview on the solar neutrino spectrum (Sec. 1), the MSW-LMA oscillation scenario (Sec. 2) and the past achievements of solar neutrino experiments (Sec.3), the recent lowthreshold analyses of ${ }^{8} \mathrm{~B}-v$ 's of SNO and Borexino are discussed in the context of oscillation physics (Secs. 4). The prospects for new experimental results in the near future are outlined (Sec. 5).

\section{The solar neutrino spectrum}

The Sun is the brightest natural neutrino source on Earth. The total neutrino flux of $\sim 6 \times 10^{10} \mathrm{~cm}^{-2} \mathrm{~s}^{-1}$ is dominated by low-energy pp- $v$ 's. The name derives from the basic fusion reaction $p+p \rightarrow$ $d+e^{+}+v_{e}$. It is the initial step of the pp fusion chain represented by the net reaction $4 p \rightarrow$ ${ }^{4} \mathrm{He}+2 e^{+}+2 v_{e}$, in which $26.7 \mathrm{MeV}$ of binding energy are released. The maximum energy of the pp- $v$ 's of $420 \mathrm{keV}$ is limited by the Q-value of the deuteron fusion. There are several sub-branches of the pp chain in which ${ }^{4} \mathrm{He}$ is produced by the fusion of protons with $\mathrm{He}, \mathrm{Li}$, or Be. Due to the larger binding energies involved, these neutrinos feature considerably higher energies, reaching a maximum of $18.8 \mathrm{MeV}$ in case of hep- $v$ 's. However, the rates of these reactions are lower than for the basic pp- $v$ 's. Moreover, the catalyst $\mathrm{CNO}$ cycle based on carbon, nitrogen, and oxygen provides a small contribution to the overall neutrino flux. Based on the standard solar model (SSM), the solar neutrino spectrum in Fig. 1 is derived [1].

${ }^{8} \mathrm{~B}$ - and ${ }^{7} \mathrm{Be}-\nu$ 's are the most relevant for spectroscopic measurements: The ${ }^{8} \mathrm{~B}-v$ spectrum extends to comparatively high energies $\left(E_{v}<14.6 \mathrm{MeV}\right)$, allowing the detection in kiloton-scale water Čerenkov detectors as the SNO experiment (Sect.3.1). The monoenergetic ${ }^{7} \mathrm{Be}-\nu$ 's are created in the electron capture on ${ }^{7} \mathrm{Be}, e^{-}+{ }^{7} \mathrm{Be} \rightarrow{ }^{7} \mathrm{Li}+v_{e}$ and form a low-energy line at $E=866 \mathrm{keV}$. They account for $\sim 10 \%$ of the total solar neutrino flux, and are the primary signal of the liquidscintillator experiment Borexino (Sect.3.2).

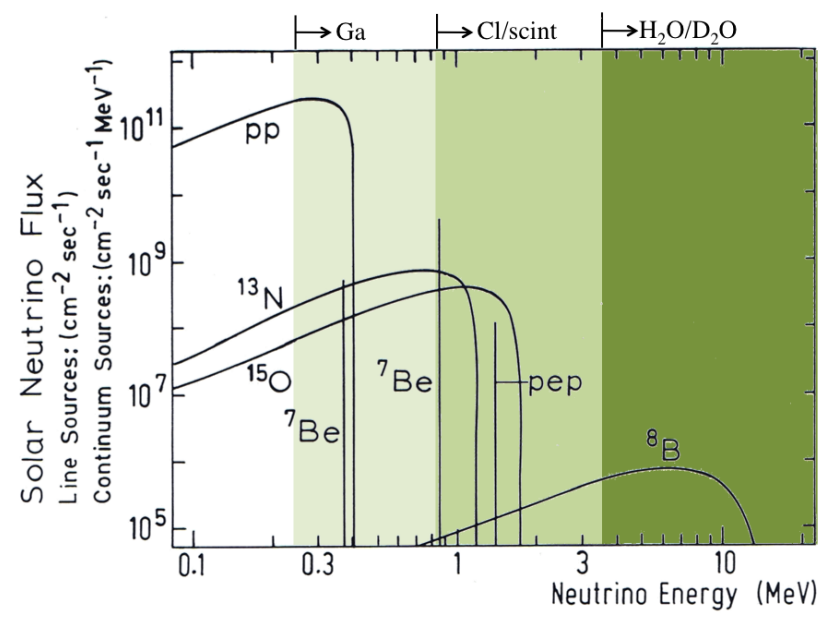

Figure 1: The solar neutrino spectrum based on the SSM [1].

\section{The MSW-LMA oscillation scenario}

Since the 1970 s, solar $v_{e}$ 's have been detected by radiochemical experiments. These detectors 
performed energy-integrated measurements of the $v$ spectrum based on the $v$-induced conversion of target nuclei to $\beta^{+}$-unstable isotopes, that were periodically extracted via their chemistry and counted by their re-decay. The Homestake, Gallex/GNO and SAGE experiments consistently reported a solar neutrino flux of about $1 / 3$ to $1 / 2$ of the theoretical SSM prediction [2, 3, 4]. While initially astrophysical explanations were considered, spectrally resolved measurements of solar and atmospheric neutrinos by Water Čerenkov detectors in the 1990s confirmed the deficit, pointing towards a non-standard behavior of the neutrinos themselves. Around 2000, the combined results of the Super-Kamiokande, SNO, and KamLAND experiments singled out neutrino flavor oscillations as cause for the missing $v_{e}$ 's.

Solar neutrinos are created as $v_{e}$, but part of them converts their flavor to $v_{\mu}$ or $v_{\tau}$ on their way to Earth. A simplified picture of two-flavor oscillations $\left(v_{e} \leftrightarrow v_{\mu}\right)$ can be applied, in which the $v_{e}$ survival probability $P_{e e}$ is given by:

$$
P_{e e}=1-\sin ^{2}\left(2 \theta_{12}\right) \sin ^{2}\left(\Delta m_{12}^{2} L / E\right) .
$$

The oscillation probability is given by the mixing angle $\theta_{12}=(34.5 \pm 1.4)^{\circ}$ [5]. The oscillation length depends on the mass-squared differences $\Delta m_{12}^{2}=(7.7 \pm 0.2) \times 10^{-5} \mathrm{eV}^{2}$ and the neutrino energy $E$. $L$ describes the oscillation baseline. However, the individual flavor states lose coherence long before reaching detectors on Earth, resulting in a distance-independent average survival probability of $\left\langle P_{e e}\right\rangle=1-0.5 \sin ^{2}\left(2 \theta_{12}\right) \approx 0.56$.

While the energy regime below $1 \mathrm{MeV}$ is well described by this large-mixing angle (LMA) solution, the interaction of neutrinos with solar matter results in a decrease of $P_{e e}$ for higher energies. As $v_{e}$ feature a larger cross-section for electron scattering than $v_{\mu}$, the large matter densities encountered in the solar center effectively increase the mass of $v_{e}$ 's beyond the mass of $v_{\mu}$ 's. Traveling through the decreasing solar matter potential, the $v_{e}$ 's undergo a resonant conversion to $v_{\mu}$, emerging from the Sun mainly as $v_{\mu}$ 's. The effect is named after its discoverers Mikheyev, Smirnov and Wolfenstein (MSW) [6].

Consequently, a distinctive energy-dependence is expected for $P_{e e}$ in the MSW-LMA scenario: At low energies $(E<1 \mathrm{MeV})$, oscillations are well described by vacuum oscillations and $P_{e e} \approx 0.56$, while for high energies $(E>5 \mathrm{MeV})$, the MSW-effects decreases the survival probability to $P_{e e}=$ $\sin ^{2} \theta_{12} \approx 0.32$. In the intermediate region, a continuous transition of $P_{e e}$ is expected (s. Fig. 3).

\section{Solar neutrino experiments}

As most solar neutrino experiments are primarily or exclusively sensitive to $v_{e}, P_{e e}$ is determined by the comparison of the measured $v_{e}$ flux to the SSM expectations. Spectrally resolved measurements have been accomplished by water Čerenkov and more recently by liquid-scintillator experiments. For both, the primary detection channel is neutrino-electron scattering (ES). The cross-section of $v_{e}$ for ES is about a factor of five larger than that of $v_{\mu, \tau}$.

In spite of the large target masses, the detected neutrino interaction rates are as low as 0.5 counts per day and ton of material due to the extremely small cross sections of weak interaction [7]. To discern this minute signal from the background of natural radiation, neutrino experiments rely on a combination of passive and active shielding as well as on the selection of low-radioactivity 
components: As the background rate created by cosmic rays on the Earth's surface is overwhelming, experiments are installed in underground laboratories in typical depths of $1-2 \mathrm{~km}$. The target liquids are purified to suppress internal backgrounds due to the $\alpha$-and $\beta$-decays of dissolved radionuclides. The radiopurity of detector materials increases in shells towards the target: As depicted in Fig. 4 for the case of Borexino, several shells of shielding liquid surround the target, absorbing $\gamma$ rays from the photosensors and the mechanical steel structure.

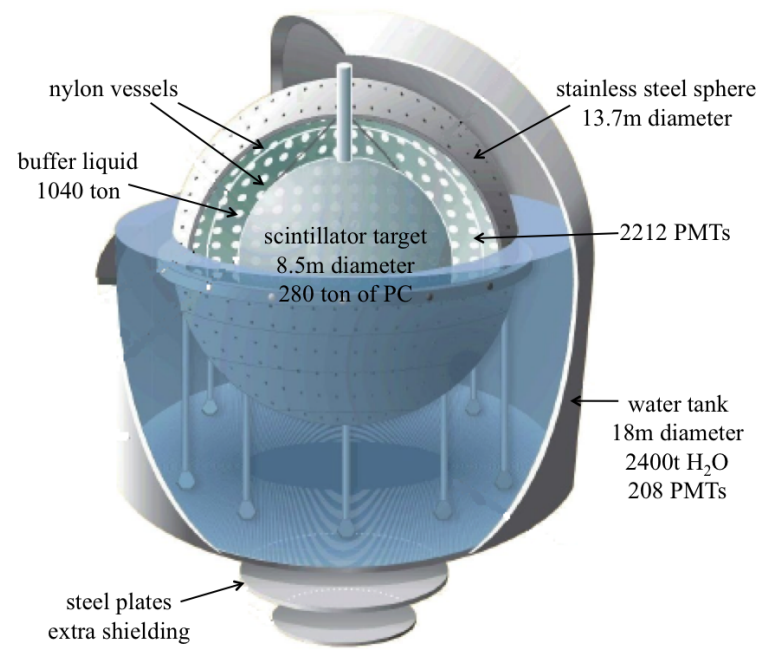

Figure 2: Layout of the Borexino experiment.

\subsection{The Sudbury Neutrino Observatory (SNO)}

The SNO experiment was running from 1999 to 2006 in an underground cavern adjacent to the Canadian Sudbury mine in a depth of $2 \mathrm{~km}$ (6000 mwe) [8]. The target consisted of 1000 tons of heavy water $\left(\mathrm{D}_{2} \mathrm{O}\right)$, contained in an acrylic sphere of $12 \mathrm{~m}$ diameter, sustained by ropes in a pool of light water filling the cavern. The Čerenkov light emitted by recoil electrons was registered by 9438 8"-photomultiplier tubes (PMTs) mounted to a support structure that surrounds the sphere at a distance of $2.9 \mathrm{~m}$. The resulting photoelectron (pe) yield was $\sim 9$ pe per $\mathrm{MeV}$ of deposited energy. The initial detection threshold was $5 \mathrm{MeV}$, limiting the analysis to ${ }^{8} \mathrm{~B}-\nu$ 's [9].

In addition to ES, the deuterons of the heavy water target offered two further detection channels: The charged-current (CC) interaction $v_{e}+d \rightarrow p+p+e^{-}$that is sensitive to $v_{e}$ only, and the neutral-current (NC) reaction $v_{x}+d \rightarrow p+n+v_{x}$ that is undergone by all neutrino flavors at equal cross sections. The flux measured in the NC reaction was $\left(5.09_{-0.43}^{+0.44} s t a t_{-0.43}^{+0.46} s y s t\right) \times 10^{6} \mathrm{~cm}^{-2} \mathrm{~s}^{-1}$, demonstrating that the total solar ${ }^{8} \mathrm{~B}-v$ flux corresponded to SSM predictions. The CC reaction returned a flux of $\left(1.76 \pm 0.05_{\text {stat }} \pm 0.09_{\text {syst }}\right) \times 10^{6} \mathrm{~cm}^{-2} \mathrm{~s}^{-1}$, corresponding to $P_{e e} \approx 0.35$ as predicted by the MSW-LMA solution [9].

\subsection{The Borexino Experiment}

The liquid-scintillator detector Borexino started operation in May 2007 at the Gran Sasso National Laboratories (LNGS) in the Italian Abruzzi (3800 mwe) [10]. Fig. 4 shows a schematic view of the detector: The target volume consists of 270 tons of the aromatic solvent pseudocumene 
(PC), doped with the wavelength shifter PPO. The liquid is contained in an ultra-thin nylon sphere of $8.5 \mathrm{~m}$ diameter, separating it from a non-scintillating PC buffer that serves as shielding from external radioactivity. This inner detector is contained in a stainless steel sphere of $13.7 \mathrm{~m}$ diameter, to which a total of 22128 "'-PMTs equipped with light-collecting Winston cones is mounted. The sphere is contained in a domed water tank of $18 \mathrm{~m}$ diameter and equipped with 208 outward-facing PMTs, serving as an active Čerenkov veto against cosmic muons.

The scintillation light yield of $\sim 500 \mathrm{pe} / \mathrm{MeV}$ surpasses that of the Čerenkov effect by far. Consequently, the instrumental detection threshold of $\sim 50 \mathrm{keV}$ is very low. However, the main challenge of the experiment was the reduction of radioactive contaminations in the detector center: The required radiopurity was achieved by distillation of the scintillator, the assembly of the nylon vessel under strict cleanroom conditions and a special selection of PMT materials. In the final experiment, a signal-to-background ratio of about 1:1 was reached for ${ }^{7} \mathrm{Be}-\nu$ 's, allowing a separation of $v$ electron recoils and $\beta$-decays by their spectral shape. The measured rate corresponds to a ${ }^{7} \mathrm{Be}-v$ flux of $(3.4 \pm 0.3) \times 10^{9} \mathrm{~cm}^{-2} \mathrm{~s}^{-1}$, or $P_{e e}=0.56 \pm 0.10$ in good agreement with the MSW-LMA expectation [7].

\section{Recent experimental results}

While the basic picture of solar neutrino oscillations has been confirmed by the results of SNO and Borexino, the energy-dependence of $P_{e e}$ in the MSW transition region from 1 to $5 \mathrm{MeV}$ is still undetermined. The transition curve of $P_{e e}$ is expected to be very sensitive to non-standard neutrino properties. Fig. 3 shows $P_{e e}(E)$ as predicted by the standard MSW-LMA scenario, compared to the model proposed by Friedland et al. in [11] that is based on non-standard interactions of $v_{e}$ in solar matter. The difference in $P_{e e}$ is significant.

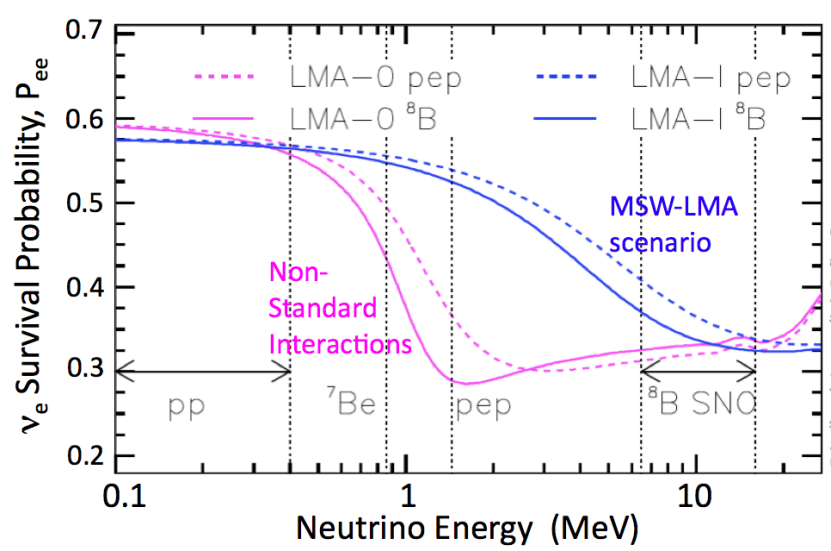

Figure 3: The solar $v_{e}$ survival probability $P_{e e}(E)$ for the standard MSW-LMA scenario and for non-standard neutrino interactions (NSI) [11]. A significant difference is discernible for the vacuum-matter transition.

Therefore, spectral measurements of the $v$ flux in the $1-5 \mathrm{MeV}$ region are very interesting: In 2010, two analyses have been published that lowered the ${ }^{8} \mathrm{~B}-v$ detection threshold below $5 \mathrm{MeV}$. The SNO low energy threshold analysis (LETA) re-evaluated the data of the first two phases of the experiment ${ }^{1}$ [12]. By a careful study of signal and background spectra, it was possible to

\footnotetext{
${ }^{1}$ In phase II, salt was dissolved in the $\mathrm{D}_{2} \mathrm{O}$ target to enhance the neutron capture signal following the NC reaction.
} 
extend the threshold to $3.5 \mathrm{MeV}$. While the results above $5 \mathrm{MeV}$ are in good agreement with the MSW-LMA scenario, the $v_{e}$ rate measured below $3.5 \mathrm{MeV}$ was low compared to the standard predictions. However, both systematic and statistical uncertainties are large, creating only a mild strain to MSW-LMA predictions [12].

The second analysis was performed by Borexino: Based on the high light yield and ultralow background conditions of the detector, a threshold of $3 \mathrm{MeV}$ could be achieved for ${ }^{8} \mathrm{~B}-\nu$ 's [13]. Fig. 4 shows the visible energy spectrum above $3 \mathrm{MeV}$ : Cosmic muons are the dominating background component. Relying on the outer muon veto and scintillator pulse shape discrimination, the event rate is reduced by more than one order of magnitude. A fiducial volume cut to the innermost 100 ton of scintillator removes the external $\gamma$ background. Short-lived cosmogenic radioisotopes induced by muon spallation in the scintillator (e.g. ${ }^{10} \mathrm{C},{ }^{12} \mathrm{~B}$ ) as well as radioisotopes of the radon chains dissolved in the liquid can be rejected based on time-correlated signals (e.g. ${ }^{214} \mathrm{Bi} / \mathrm{Po}$-coincidences, or ${ }^{212} \mathrm{Bi} / \mathrm{Po}$-coincidences to remove ${ }^{208} \mathrm{Tl}$ by statistical subtraction, see [13] for details).

The observed rate above $5 \mathrm{MeV}$ corresponds to an ${ }^{8} \mathrm{~B}-v$ flux of $(2.7 \pm 0.4 \pm 0.2) \times 10^{6} \mathrm{~cm}^{-2} \mathrm{~s}^{-1}$ [13], in good agreement with earlier SNO and Super-Kamiokande ES results [9, 14]. Including the range to $3 \mathrm{MeV}$, a flux of $(2.4 \pm 0.4 \pm 0.1) \times 10^{6} \mathrm{~cm}^{-2} \mathrm{~s}^{-1}$ is measured [13], low but not in conflict with standard MSW-LMA predictions.

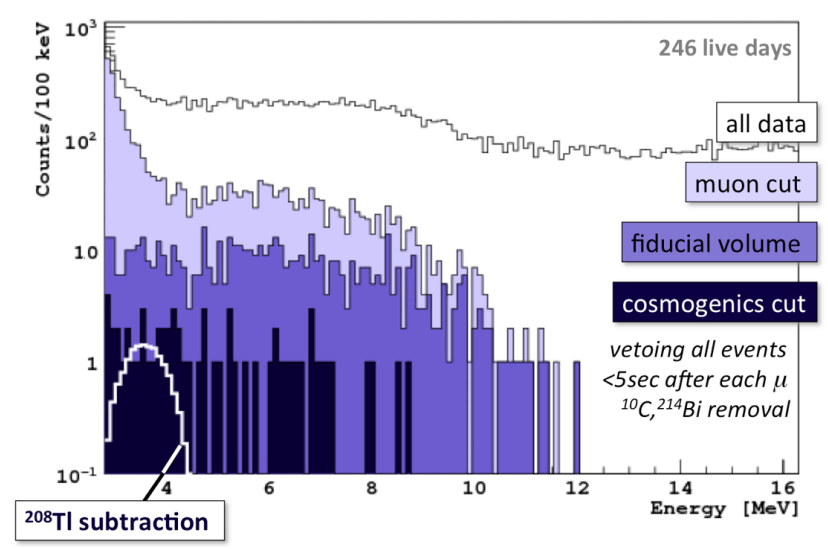

Figure 4: The visible energy spectrum of Borexino above $3 \mathrm{MeV}$. Several cuts on background have to be applied to extract the ${ }^{8} \mathrm{~B}-v$ electron recoil spectrum [13].

\section{Future prospects}

In the light of this new results, a further exploration of the MSW transition region seems very interesting due to its potential to reveal new non-standard neutrino physics. A low-threshold analysis of Super-Kamiokande ${ }^{8} \mathrm{~B}$ data is currently on-going. Borexino is expected to publish further data in the near future: The current accuracy of the ${ }^{7} \mathrm{Be}-v$ flux measurement of $\sim 10 \%$ is going to be reduced to $5 \%$ or lower. This improvement relies on increased statistics and a dedicated calibration campaign: Based on the insertion of radioactive calibration sources in the scintillator, the uncertainties of spacial and energy reconstruction can be reduced. Moreover, a direct measurement of the pep- $v$ line at $1.44 \mathrm{MeV}$ seems within reach: The dominant background 
arises from the $\beta^{+}$-decays of cosmogenic ${ }^{11} \mathrm{C}$ in the scintillator, surpassing the pep- $v$ rate by about an order of magnitude. However, an event-by-event subtraction of this background is possible by a triple-coincidence using the initial muon, the knock-out neutron from ${ }^{12} \mathrm{C}$ and the ${ }^{11} \mathrm{C}$ decay signal [15]. The ${ }^{11} \mathrm{C}$ reduction might also allow the direct detection of CNO- $v$ 's. The $\mathrm{CNO}$ contribution to solar energy production is poorly known and also closely linked to solar metallicity [16].

The SNO experiment stopped operation in 2006. However, there are plans to replace the $\mathrm{D}_{2} \mathrm{O}$ target with liquid scintillator. The target mass of the resulting $\mathrm{SNO}+$ detector will be three times larger than in Borexino, while the exceptional depth will reduce cosmogenic backgrounds considerably, creating excellent conditions for pep/CNO- $v$ detection [17].

\section{Conclusions}

Over the last decade, the experimental results on solar neutrinos have established the MSWLMA oscillation scenario. While vacuum and matter-dominated oscillation regimes are now well determined, the transition region between 1 and $5 \mathrm{MeV}$ might still reveal new, non-standard neutrino properties. Both SNO and Borexino have already explored part of this energy regime by lowering the threshold for ${ }^{8} \mathrm{~B}-\nu$ detection. While the results are not yet conclusive, new results of Borexino on ${ }^{7} \mathrm{Be}$ and pep- $v$ 's are expected for the near future.

\section{References}

[1] J. N. Bahcall et al., Astrophys. J. Suppl. 165, 400-431 (2006), astro-ph/0511337.

[2] R. Davis, Prog. Part. Nucl. Phys., 32, 13-32 (1994).

[3] GNO collaboration, Phys. Lett. B, 616, 174-190 (2005), hep-ex/0504037.

[4] SAGE collaboration, Phys. Rev. C, 80, 015807 (2009), nucl-ex/0901.2200.

[5] M. C. Gonzalez-Garcia, M. Maltoni, Phys. Rept. 460, 1 (2008), hep-ph/0704.1800.

[6] Q. Y. Liu et al., Commun. Theor. Phys. 44, 505 (2005), hep-ph/0509182.

[7] Borexino collaboration, Phys. Rev. Lett. 101, 091302 (2008), astro-ph/0805.3843.

[8] SNO collaboration, Nucl. Instrum. Meth. A 449, 172 (2000), nucl-ex/9910016.

[9] SNO collaboration, Phys. Rev. Lett. 89, 011301 (2002), nucl-ex/0204008

[10] Borexino collaboration, Nucl. Instrum. Meth. A 660, 568 (2009), physics.ins-det/0806.2400.

[11] A. Friedland et al., Phys. Lett. B, 594, 347-354 (2004), hep-ph/0402266.

[12] SNO collaboration, Phys. Rev. C, 81, 055504 (2010), nucl-ex/0910.2984.

[13] Borexino collaboration, Phys. Rev. D, 82, 033006 (2010), astro-ph/0808.2868.

[14] Super-Kamiokande collaboration, Phys. Rev. D, 78, 032002 (2008), hep-ex/0803.4312.

[15] C. Galbiati et al., Phys. Rev. C, 71, 055805 (2005), hep-ph/0411002.

[16] C. Pena-Garay, A. Serenelli (2008), astro-ph/0811.2424.

[17] SNO+ collaboration, Prog. Part. Nucl. Phys. 57, 150-152 (2006). 